\title{
Otoliths Identifiers Using Image Contours EFD
}

\author{
R. Reig-Bolaño, Pere Marti-Puig, S. Rodriguez, J. Bajo, \\ V. Parisi-Baradad, and A. Lombarte
}

\begin{abstract}
In this paper we analyze the characteristics of an experimental otolith identification system based on image contours described with Elliptical Fourier Descriptors (EFD). Otoliths are found in the inner ear of fishes. They are formed by calcium carbonate crystals and organic materials of proteic origin. Fish otolith shape analysis can be used for sex, age, population and species identification studies, and can provide necessary and relevant information for ecological studies. The system we propose has been tested for the identification of three different species, Engraulis encrasicholus, Pomadasys incisus belonging to the different families (Engroulidae and Haemolidae), and two populations of the species Merluccius merluccius (from CAT and GAL) from the family Merlucciidae. The identification of species from different families could be carried out quite easily with some simple class identifiers -i.e based on Support Vector Machine (SVM) with linear Kernel-; however, to identify these two populations that are characterized by a high similarity in their global form; a more accurate, and detailed shape representation of the otoliths are required, and at the same time the Otolith identifiers have to deal with a bigger number of descriptors. That is the principal reason that makes a challenging task both the design and the training of an otolith identification system, with a good performance on both cases.
\end{abstract}

Keywords: Otoliths Analysis, Elliptic Fourier Descriptors (EFD), Shape Analysis, Image Processing, Pattern Recognition, Pattern Classification.

R. Reig-Bolaño and Pere Marti-Puig

Department of Digital Information and Technologies, University of Vic (UVIC)

C/ de la Laura, 13, E-08500, Vic, Catalonia, Spain

e-mail: ramon.reig@uvic.cat, pere.marti@uvic.cat

S. Rodriguez and J. Bajo

BISITE Group (USAL), Pza. de la Merced s/n, 370008 Salamanca, Spain

V. Parisi-Baradad

Department of Electronic Engineering, Politechnical Univerity of Catalonia (UPC)

A. Lombarte

ICM-CSIC, Passeig Marítim 37-49, 08003, Catalonia, Spain

A.P. de Leon F. de Carvalho et al. (Eds.): Distrib. Computing \& Artif. Intell., AISC 79, pp. 9-16. springerlink.com

(C) Springer-Verlag Berlin Heidelberg 2010 


\section{Introduction}

Otoliths are found in the inner ear of fishes. They are formed by calcium carbonate crystals and organic materials of proteic origin. The shapes and proportional sizes of the otoliths vary with fish species. In general, fish from highly structured habitats such as reefs or rocky bottoms will have larger otoliths than fish that spend most of their time swimming at high speed in straight lines in the open ocean. Flying fish have unusually large otoliths, possibly due to their need for balance when launching themselves out of the water to "fly" in the air. Often, the fish species can be identified from distinct morphological characteristics of an isolated otolith. It is also widely accepted that fish otolith shape analysis can be used for sex, age, population and species identification studies, and can provide necessary and relevant information for ecological studies. Successful stock discriminations using otolith shape analyses have been reported by Casselman et al. (1981), Bird et al. (1986), Campana \& Casselman (1993). Moreover the variability in the left sagitta otolith shape has been related to genetic, ontogenetic and environmental factors (Lombarte et al. 2003). Several methods are used to describe and compare form in morphological studies, such as ratios of linear dimensions, Euclidean distance matrix analysis, eigenshape analysis, and several variations of Fourier analysis (Chen et al. 2000; Iwata 2002). The traditional approach to contour feature extraction is based on expanding the contour into a two-dimensional series by means of elliptic Fourier analysis (Kuhl and Giardina 1982). The data are reduced by selecting only a set of coefficients for the expansion. Appropriate feature extraction and compaction is essential for obtaining good results in automatic classification systems. One of the major problems when complex data sets are classified is the number of variables involved. In pattern recognition and image processing, feature extraction is a form of dimensionality reduction. Although Elliptic Fourier Descriptors (EFD) can represent any outline when a large number of coefficients are used, in practical applications the number of harmonics is limited to a certain value, i.e 40 coefficients (Parisi et al. 2005; Tracey et al. 2006); therefore, EFDs only represent an approximation to the original contour. This problem comes to light when there is a close similarity between different species or populations from the same species. In these cases the design and the training of the identifier is a challenging task. In this paper we will show some results on both cases.

\section{Materials and Methods}

The test material was taken from the AFORO database (http://aforo.cmima.csic.es), a web based environment for shape analysis of fish otoliths (Lombarte et al. 2006). The database is regularly updated and at present $(05 / 20 / 2010)$ it contains a total of 2874 high resolution images corresponding to 841 species and 168 families from the Western Mediterranean Sea, Weddell Sea and Antartic Peninsula, Southwestern Atlantic (Uruguay and Argentina, Northwestern Atlantic and Gulf of Mexico), Northeastern Atlantic (From Senegal to North Sea), Southeastern Atlantic (Namibia), Indic (Tanzania) and Pacific (New 
Caledonia, Alaska, Canada, Perú, and Chile). The sagitta is the otolith with the largest morphologic variability and therefore is the most studied. This database provides an open online catalogue of otolith images, and is associated with a shape analysis module that uses pattern recognition techniques and applies Fourier transform (FT), curvature scale space (CSS) and wavelet transform (WT). The site also implements a search in an identification engine, using query images of otoliths. The method presented in this paper has been tested with otoliths from three different species, Engraulis encrasicholus, Pomadasys incisus belonging to the different families (Engroulidae and Haemolidae), and two populations of the species Merluccius merluccius (from CAT and GAL) from the family Merlucciidae. These groups are characterized by having high similarity between species; therefore, detailed shape analyses of their otoliths can help to identify and discriminate morphologically close species. But, at the same time, this makes the identifier more difficult to design and train.

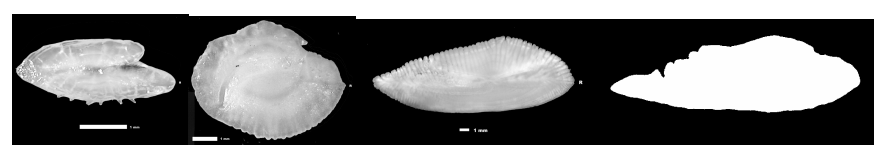

Fig. 1 (from left to right) Left sagitta Otoliths from Engraulis encrasicholus, Pomadasys incisus, Merluccius merluccius CAT (Catalonia) and Merluccius merluccius CAN (Canada)

The identifiers are based on a simple Support Vector Machine (SVM) with a linear kernel, like the one described in the tutorial of Burges (1998), trained for each one of the classes we want to classify, the output of the system will be one of the following classes: ENG -Engraulis-, POM -Pomadasys-, CAT -Merluccius CAT-, GAL -Merluccius GAL-, NOT -not determinate-. The output will be decided from the binary outputs of the SVMs. The descriptors used in the identifiers will be obtained from the EFD of the image contour under test.

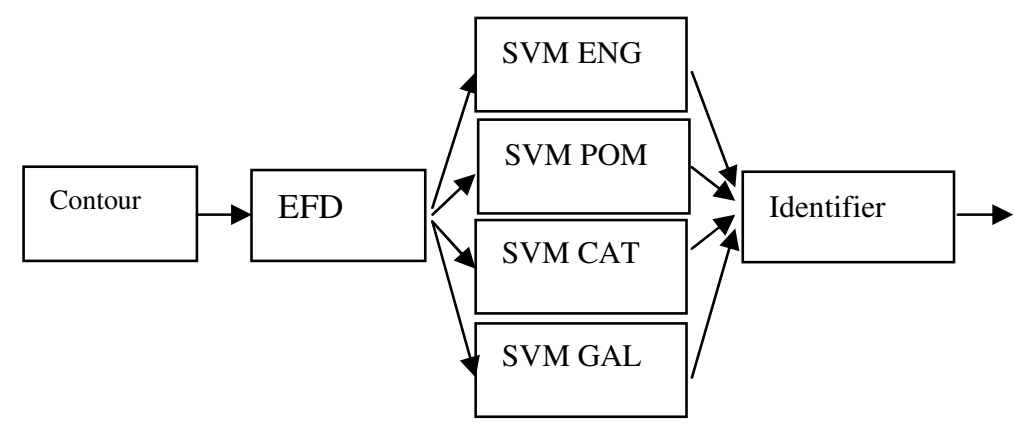

Fig. 2 Schema for the identification system studied in this paper. In some cases de EFD analysis would be independent for each SVM. 


\subsection{Contours Extraction and Elliptic Fourier Descriptors (EFD)}

The first step of the system following the schema of Fig. 2 is the contour extraction from the otolith images. In AFORO database all the images are highly contrasted respect to the background, so any contour extractor works properly; in our case we have used a morphological contour extractor (Serra 1982); it is applied on a segmented binary image like the last one that is represented in Fig.1, and the result is shown in Fig 3.
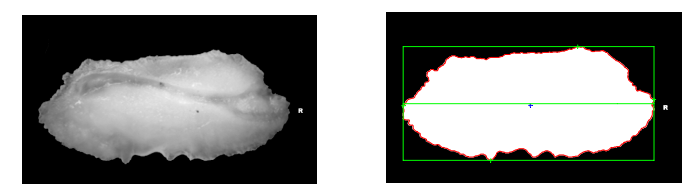

Fig. 3 Otolith contour extraction from a normalized image. Normalized otolith image (left panel) with its contour (in red) inside an enclosing rectangular box (right panel).

An elliptical Fourier function perfectly describes a closed curve with an ordered set of data points in a two-dimensional plane. The function decomposes the curve orthogonally into a sum of harmonically related ellipses (Kuhl and Giardina 1982). These ellipses can be combined to reconstruct an arbitrary approximation to the closed curve.

A closed contour with period $T$ is defined by the evolution of its coordinates $x(t)$ and $y(t)$ along the variation $t$ within the length of this contour, and can be expanded as follows:

$$
x(t)=a_{0}+\sum_{n=1}^{\infty}\left[a_{n} \cos \left(\frac{2 \pi n t}{T}\right)+b_{n} \sin \left(\frac{2 \pi n t}{T}\right)\right] y(t)=c_{0}+\sum_{n=1}^{\infty}\left[c_{n} \cos \left(\frac{2 \pi n t}{T}\right)+d_{n} \sin \left(\frac{2 \pi n t}{T}\right)\right]
$$

The coefficients $a_{n}, b_{n}, c_{n}$ and $d_{n}$ are an alternative way of perfectly describing the contour. The signal is characterized and the information reduced by selecting a reduced set of $4 \mathrm{~N}$ coefficients, which leads to the expression:

$$
x_{N}(t)=a_{0}+\sum_{n=1}^{N}\left[a_{n} \cos \left(\frac{2 \pi n t}{T}\right)+b_{n} \sin \left(\frac{2 \pi n t}{T}\right)\right] y_{N}(t)=c_{0}+\sum_{n=1}^{N}\left[c_{n} \cos \left(\frac{2 \pi n t}{T}\right)+d_{n} \sin \left(\frac{2 \pi n t}{T}\right)\right]
$$

Given a contour obtained from an image, the coefficients $a_{n}, b_{n}, c_{n}$ and $d_{n}$ can be calculated as proposed by Kuhl and Giardina (1982) for connectivity -8 and simplified (Abidi and Gonzalez 1986) for connectivity -4 .

At this point it is important to note that $x_{N}(t)$ and $y_{N}(t)$ only represent an approximation to the original contour that improves as $N$ increases. When a particular value of $N$ is chosen, it can be assumed that with $4 N+2$ parameters the resulting approximation is a valid characterization of the original contour. To use EFDs effectively, otolith images need to be normalized in order to have the same image acquisition conditions. The otolith contour is usually normalized for size and orientation: the coefficients $a_{0}$ and $c_{0}$ are taken as 0 (this locates the centre of mass at the origin), the rest of the coefficients are normalized so that $a_{1}=1$, and the main radial is normalized to 1 . 
To complete the EFD computation, we obtain the Fourier series expansion of one period T' (of K points) of the discrete periodical functions $y(k)$ and $x(k)$. Taking into account that the range of the digital frequencies $\left(\omega_{n}\right)$ that can be represented in the discrete domain is limited to the set of harmonics given by the expression (Proakis 2007), then the discrete Fourier series expansion of $y(k)$ takes the form:

$$
\begin{gathered}
\omega_{n}=\frac{2 \pi}{T^{\prime}} n=\frac{2 \pi}{K} n \quad n=0, . ., K-1 \\
y(k)=c_{0}+\sum_{n=1}^{K}\left[c_{n} \cos \left(\frac{2 \pi}{K} n k\right)+d_{n} \sin \left(\frac{2 \pi}{K} n k\right)\right] \quad \begin{array}{l}
k=0, \ldots, K-1 \\
n=0, \ldots, K-1
\end{array}
\end{gathered}
$$

The $y_{N}$ approximation is carried out by selecting a reduced set of $2 N$ coefficients, with $N<K$ :

$$
y_{N}(k)=c_{0}+\sum_{n=1}^{N}\left[c_{n} \cos \left(\frac{2 \pi}{K} n k\right)+d_{n} \sin \left(\frac{2 \pi}{K} n k\right)\right] \begin{gathered}
k \\
=0, . ., K-1 \\
n=1, . ., N
\end{gathered}
$$

The coefficients of the one-dimensional Fourier series can be obtained very quickly with the FFT (Fast Fourier Algorithm). The complex values provided by the FFT are a compact representation of the two real coefficients given in the Fourier series expansion (Cooley and Tukey 1965). And the same could be done for the $x(k)$ function.

\subsection{Support Vector Machine (SVM) and Identifier}

The identifiers are based on a set of SVM with linear kernel, like the one described in the tutorial of Burges (1998). During the training phase, the linear Kernel (eq. 8) measures the Euclidean distance between the annotated descriptors $S_{I}$ and $S_{J}$, and finds the optimal $\alpha_{l}$ set values to separate one class from the others, which corresponds to the selection of an hyperplane in the L-dimensional space of the descriptors, to separate one class from the others.

$$
K\left(S_{I}, S_{J}\right)=\sum_{l e L} \alpha_{l} d_{l}\left(S_{I}, S_{J}\right)
$$

The design point from our approach was the selection of the descriptors used in the training and subsequently into the test phase.

Once we have trained each one of the SVM corresponding to the classes, during the test phase the Identifier is fed with those results, and the output of the system should be one of the following classes: ENG -Engraulis-, POM Pomadasys-, CAT -Merluccius CAT-, GAL -Merluccius GAL-, NOT -not determinate-. In the case that more than one is activated, the output would be NOT, except for the CAT and GAL case, as they correspond to the same species.

\section{Implementation and Results}

This experimental Identification system has been implemented with Octave 3.2 software under Linux 10.04 on a Core 2 Duo T7100 / 1.8 GHz Laptop with 2 GB 
RAM. We used a DATASET extracted from AFORO database: 10 specimens of Engraulis encrasicholus, from the family Engroulidae, 14 specimens of Pomadasys incisus belonging to the family Haemolidae, and 44 specimens from different populations of Merluccius merluccius from the family Merlucciidae. The database is available from http://aforo.cmima.csic.es for research purposes. For the training step corresponding to the SVM of ENG, POM, CAT, GAL we divide and annotate the population with 10 specimens of each group. During the training we select randomly $50 \%$ of this population, with a minimum of $20 \%$ from the corresponding class. The test phase was done using the complete population. In all the cases we use a retraining phase with part of the training individuals.

The main parameters analyzed for the design of the system are two: the number of points of the contours $(\mathrm{K})$, it is used to normalize the contours of different specimens. The value K could go from 2 to 1024, with K very low we only represent values related to basic diameters of the contour. But with $\mathrm{K}$ getting high, the number of parameters increases. Another value is $\mathrm{L}$ the total number of dimensions on the classification space, it is related with the order of the EFD (N), with the equation $\mathrm{L}=4 \cdot(2 \cdot \mathrm{N})-2$, moreover it is necessary that $\mathrm{N} \leq \mathrm{K} / 2$. With $\mathrm{L}$ low, the contour is represented with basic ellipsoids, not having into account the detail of the contours, but at the same time, the classifiers have a low dimensional space (easier to train and generalize). When $\mathrm{L}$ goes higher, the contour is represented with more details, however, the classifier has higher dimension and becomes instable and sometimes could not be generalized.

The plotted results are for medium values of $\mathrm{K}=64$, and $\mathrm{N}=32$, which leads to a decision space of $\mathrm{L}=254$ parameters. To represent the results we plot the true
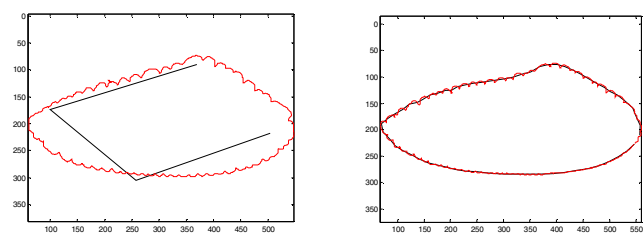

Fig. 4 Results for CAT contour (in red), respect to the undersampled contour (in black) with $\mathrm{K}=4$ and $\mathrm{N}=2$ in the left panel, and the same with $\mathrm{K}=64$ and $\mathrm{N}=32$ in the right one.
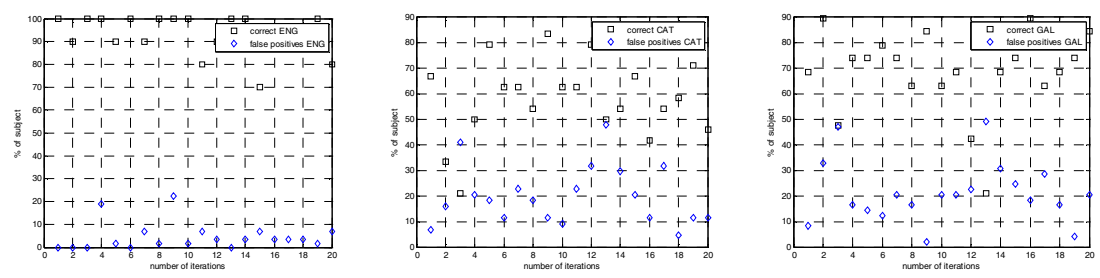

Fig. 5 Results for ENG, CAT, GAL identification using $\mathrm{K}=64$ and $\mathrm{N}=32(\mathrm{~L}=254)$, the mean value of the correct identification is $93 \%, 57 \%$ and $68 \%$; The $\%$ of false positives is $4.6 \%$, $20 \%$ and $21 \%$. 

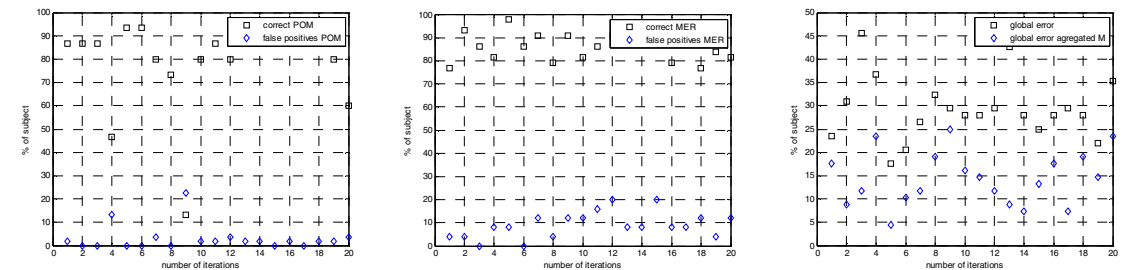

Fig. 6 From lest to right results for POM and MER identification, using $\mathrm{K}=64$ and $\mathrm{N}=32$ $(\mathrm{L}=254)$, the mean value of the correct identification is $79 \%$ and $86 \%$ and the false positives is $3 \%$ and $9 \%$. Results for Global error identification (the right panel) we compare the error without aggregating CAT and GAL, and with their aggregation as MER. The mean error is $29 \%$ in one case and $14 \%$ in the other

classifications results (in \%), represented with black squares; and in the same plot we draw the value of false postitives for each one of the classes (in \%), represnted by blue romboids.

The results for CAT and GAL have a great number of false positives, as there is a great similarity between the two populations of the same specie. If we aggregate the results as the specie Merluccius merluccius (MER), the results are similar to the other species, as we can see in Fig 6.

\section{Discussion}

For the same $\mathrm{K}=64$, we have compared the results for $\mathrm{N}=8,16$ and are quite similar, although the CAN, GAL errors become greater, for $\mathrm{N}=4(\mathrm{~L}=30)$ the global error reaches $51 \%$ without aggregation and $29 \%$ with aggregation. Meaning that the class classification could be acceptable with a low number of descriptors, but the population cannot. There is a somehow surprising result if we maintain $\mathrm{N}=4(\mathrm{~L}=30)$ but $\mathrm{K}=16$, then the results are better, global error $30 \%$ and $17 \%$, and the partial result similar to those of the section 4 , and the same happens with $K=8$. These could be seen as an effect of having more frequencies information in less coefficients as the number of points in the contour decreases; however, the main difference with initial values $\mathrm{K}=64$ is that not all the trains are valid, and the standard deviation of the measures are greater in all the graphics. With $\mathrm{K}=4$ and $\mathrm{N}=2(\mathrm{~L}=14)$, the global errors are $36 \%$ and $16 \%$ respectively but the identification errors are greater in more training essays. With $\mathrm{K}=2$ and $\mathrm{N}=1$ we have a lot of errors. With $\mathrm{K}=128$ or greater, the system becomes more difficult to train and the system gets worse results. Another interesting point is the results for the classification of populations, but they have to be more accurately studied, in deeper detail with a wider set of otoliths, and selecting by hand the best classifiers of the training set.

\section{Conclusion}

The proposed method for otoliths identification could be a good method analyzing these preliminary results. But to be used as a classification method in an automatic 
system as an extension to the AFORO database, it must be generalized to more than 800 species, some with differentiated populations, and it must be designed and extensively tested with all the database of more than 2800 otoliths and growing up every day. In this experiment the detail of the contour was not significant, but in this new and open scenario that should not be always true, then it must be seen if the system could work for $\mathrm{K}>128$ and $\mathrm{N}>64$. With this experiment we have completed an identification system for fish otoliths, and our next work would be to improve its performance, tuning each one of the elements and analyzing alternative solutions for all of them.

Acknowledgements. This work has been supported by 'Ajuts per a estades de recerca fora de Catalunya 2009-10' and the Digital Technologies Research Group from the University of Vic: cost center R008-R0904.

\section{References}

Abidi, M.A., Gonzalez, R.C.: Shape Decomposition Using Elliptic Fourier Descriptors. In: Proc. 18th IEEE Southeast Sympo. Sys. Theory, Knoxvlle, TN USA, pp. 53-61 (1986)

Bird, J.L., Eppler, D.T., Checkley, D.M.: Comparisons of herring otoliths using Fourierseries shape-analysis. Can. J. Fish. Aquat. Sci. 43(6), 1228-1234 (1986)

Burges, C.: A Tutorial on Support Vector Machines for Pattern Recognition. Data Mining and Knowledge Discovery 2, 121-167 (1998)

Campana, S.E., Casselman, J.M.: Stock discrimination using otolith shape analysis. Can. J. Fish. Aquat. Sci. 50, 1062-1083 (1993)

Casselman, J.M., Collins, J.J., Crossman, E.J., Ihssen, P.E., Spangler, G.R.: Lake whitefish (Coregonus clupeaformis) stocks of the Ontario waters of Lake Huron. Can. J. Fish. Aquat. Sci. 38, 1772-1789 (1981)

Chen, S.Y.Y., Lestrel, P.E., Kerr, W.J.S., McColl, J.H.: Describing shape changes in the human mandible using elliptical Fourier functions. Eur. J. Orthodont. 22, 205-216 (2000)

Cooley, J.W., Tukey, J.W.: An Algorithm for the Machine Calculation of Complex Fourier Series. Math. Computat. 19, 297-301 (1965)

Iwata, H., Ukai, Y.: SHAPE: a computer program package for quantitative evaluation of biological shapes based on elliptic Fourier descriptors. J. Hered. 93, 384-385 (2002)

Kuhl, F.P., Giardina, C.R.: Elliptic Fourier features of a closed contour. Comput. Graph. Image Process. 18, 236-258 (1982)

Lombarte, A., Torres, G.J., Morales-Nin, B.: Specific Merluccius otolith growth patterns related to phylogenetics and environmental. J. Mar. Biol. Ass. U.K. 83, 277-281 (2003)

Lombarte, A., Chic, O., Parisi-Baradad, V., Olivella, R., Piera, J., García-Ladona, E.: A web-based environment for shape analysis of fish otoliths. The AFORO database. SCI. MAR. 70(1), 147-152 (2006)

Parisi-Baradad, V., Lombarte, A., García-Ladona, E., Cabestany, J., Piera, J., Chic, Ò.: Otolith shape contour analysis using affine transformation invariant wavelet transforms and curvature scale space representation. Mar. Freshw. Res. 56, 795-804 (2005)

Proakis, J.G., Manolakis, D.G.: Digital Signal Processing, 4th edn. Pearsons Education (2007)

Rosenfeld, A., Pfaltz, J.L.: Distance functions on digital pictures. Pattern Recognition 1(1), 33-61 (1968)

Serra, J.: Image Analysis and Mathematical Morphology. Academic Press, New York (1982)

Tracey, S.R., Lyle, J.M., Duhamelb, G.: Application of elliptical Fourier analysis of otolith form as a tool for stock identification. Fisheries Research 77, 138-147 (2006) 